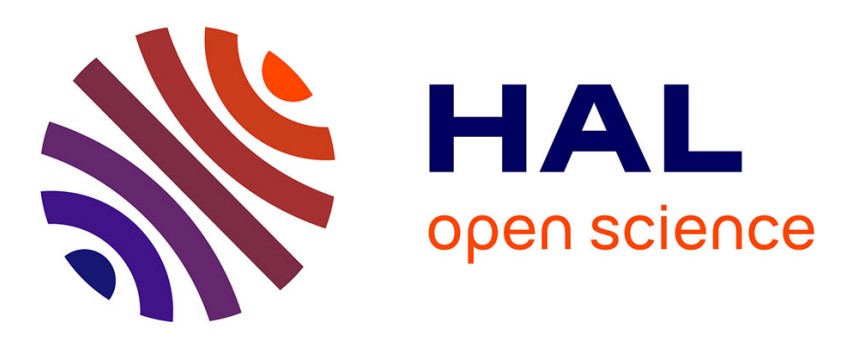

\title{
A WIDE-BAND A/D CONVERTER FOR THE SOFTWARE-DEFINED RADIO SYSTEM
}

Davud Asemani, Jacques Oksman

\section{To cite this version:}

Davud Asemani, Jacques Oksman. A WIDE-BAND A/D CONVERTER FOR THE SOFTWAREDEFINED RADIO SYSTEM. IEEE International Conference on Signal Processing and Communications, Nov 2007, Dubai, United Arab Emirates. pp.947-950. hal-00261159

\section{HAL Id: hal-00261159}

https://hal-centralesupelec.archives-ouvertes.fr/hal-00261159

Submitted on 6 Mar 2008

HAL is a multi-disciplinary open access archive for the deposit and dissemination of scientific research documents, whether they are published or not. The documents may come from teaching and research institutions in France or abroad, or from public or private research centers.
L'archive ouverte pluridisciplinaire HAL, est destinée au dépôt et à la diffusion de documents scientifiques de niveau recherche, publiés ou non, émanant des établissements d'enseignement et de recherche français ou étrangers, des laboratoires publics ou privés. 


\title{
A WIDE-BAND A/D CONVERTER FOR THE SOFTWARE-DEFINED RADIO SYSTEM
}

\author{
Davud Asemani , Jacques Oksman \\ Supelec, Department of signal processing and electronic systems \\ 3, Rue Joliot Curie, Gif-sur-Yvette, 91192, France \\ Email: firstname.lastname@Supelec.fr
}

\begin{abstract}
The HFB structure is a suitable candidate to realize a wide-band A/D convertor for the Software-Defined Radio (SDR) systems. Two MIMO Time-Division Multiplexing (TDM) and subband HFB architectures are compared with the classical one in this paper. Simulating in the timedomain, the MIMO HFBs exhibit a better performance than the classical one in terms of output resolution as well as the sensitivity to the realization errors of analysis filter bank. In opposite to the classical HFB case, the MIMO HFBs provide an LTI input-output relation. It is shown that the blind estimation and noise cancelation techniques may be used in the MIMO case for correcting the realization errors. The MIMO and classical HFBs are compared in terms of computational complexity as well.
\end{abstract}

Index Terms - A/D converter, Software-defined radio

\section{INTRODUCTION}

The demand for A/D or D/A converters with higher speeds has dramatically increased for realizing the new communications concepts such as SDR approach [1]. Nowadays, the performance of ADCs still can not fulfill the high requirement of the wide-band receiver of SDR approach. The primary target of SDR is to be compatible with various wireless communication protocols [2]. Stimulated by the need for a global communication network, SDR will form a new industry on an even larger scale than the personal computer industry [3]. The receiver and transmitter of SDR are open to a wider segment of spectrum so that the conventional analog sharp filters and channelizer are substituted by digital filtering. Then, the cost of receiver is independent of the channel number, namely, will be constant [2].

A high-speed parallel A/D conversion technique was offered employing Quadrature Mirror Filter (QMF) consisting of analysis and synthesis filter banks associated with discretetime and digital filters respectively [1]. To avoid the disadvantages of discrete-time filter bank and its realization difficulties in A/D conversion, analog filters have been offered to operate instead of discrete-time analysis filter bank. Fig. 1 illustrates the general architecture of a Hybrid Filter Bank (HFB) ADC. An $M$-branch classical HFB-based

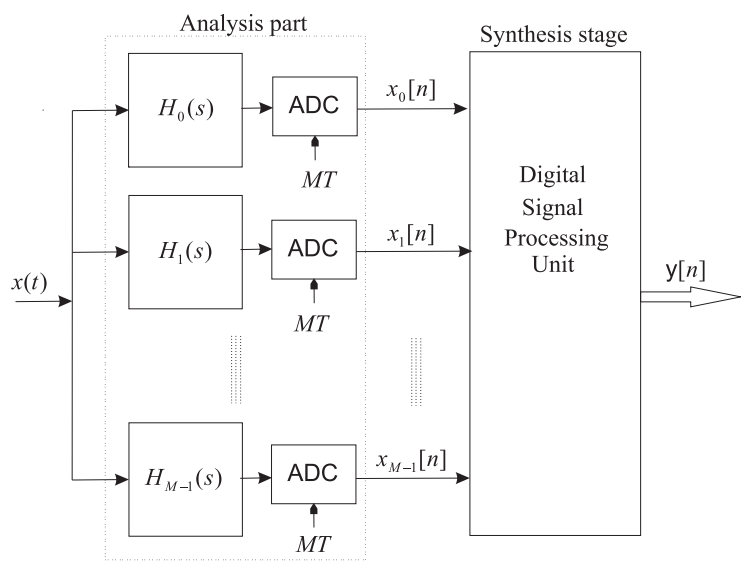

Fig. 1. General diagram of HFB-based ADC. The output $y[n]$ represent a sequence $x\left[n^{\prime}\right]$ or a vector of $M$ sequences $\widehat{\mathbf{s}}[n]$ for the classical and MIMO HFBs respectively.

ADC uses $M$ digital filters in the synthesis stage (Fig. 2) [4], [5]. The real challenge in the implementation of HFB-based A/D converters is nevertheless its high sensitivity to the realization errors [6]. In fact, a very small deviation in the analysis filter bank results in a large degradation of performance so that the respective HFB ADC would no longer be useful [5]. On the other hand, the realization errors of analog analysis filters are rarely avoidable. Digital techniques have been considered for overcoming the problem of high sensitivity to the realization errors recently [7]. Nevertheless, the proposed methods are so limited to some types of errors or to a very specific case [8]. The TDM and subband HFB architectures have been proposed so that an LTI system relates the associated input-outputs since the classical HFB is non-LTI [9], [10]. It is demonstrated that the MIMO HFB ADCs may not only exhibit less sensitivity to the realization errors, but also be corrected using the blind techniques. A complete comparison between the classical and MIMO HFB architectures is presented by the results of temporal simulations in this paper. Next section introduces both group of HFB architectures. Section III describes the design of synthesis stage of HFB. In section IV, the different HFB architectures are evaluated and compared. At last, the 


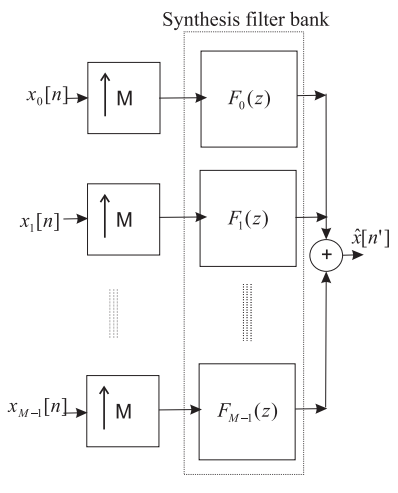

(a) Classical HFB

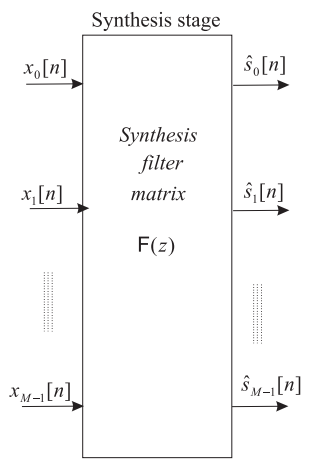

(b) MIMO HFB
Fig. 2. The synthesis stage for the classical (a) and MIMO (b) HFB architectures.

results are summarized in conclusion.

\section{PERFECT RECONSTRUCTION EQUATIONS}

\section{II-A. Classical HFB architecture}

The classical HFB-based ADC is considered (Fig. 2 (a) and 1). Neglecting the $M$ quantizers of ADCs, the spectral description $\widehat{X}\left(e^{j \omega}\right)$ of output $\widehat{x}\left[n^{\prime}\right]$ may be decomposed as following [5]:

$$
\left.\underbrace{\widetilde{X}(j \Omega) \cdot T_{\circ}\left(e^{j \omega}\right)}_{\text {Distortion part }}\right|_{\Omega=\frac{\omega}{T}}+\left.\underbrace{\sum_{m=1}^{M-1} \tilde{X}(j \Omega) \cdot T_{m}\left(e^{j \omega}\right)}_{\text {Aliasing part }}\right|_{\Omega=\frac{\omega}{T}-\frac{2 \pi}{M T} m}
$$

where $\tilde{X}(j \Omega)$ represents the periodic extension of of the input $X(j \Omega)$ considering the spectral interval $\left[-\frac{\pi}{T}, \frac{\pi}{T}\right]$ (with the period $\left.\frac{2 \pi}{T}\right)$. The distortion $T_{\circ}\left(e^{j \omega}\right)$ and aliasing $T_{m}\left(e^{j \omega}\right)$ functions $(m=1, \ldots, M-1)$ are:

$$
\left\{\begin{array}{l}
T_{\circ}\left(e^{j \omega}\right)=\frac{1}{M T} \sum_{k=0}^{M-1} F_{k}\left(e^{j \omega}\right) \cdot \widetilde{H}_{k}\left(j \frac{\omega}{T}\right) \\
T_{m}\left(e^{j \omega}\right)=\frac{1}{M T} \sum_{k=0}^{M-1} F_{k}\left(e^{j \omega}\right) \cdot \widetilde{H}_{k}\left(j \frac{\omega}{T}-j \frac{2 \pi}{M T} m\right)
\end{array}\right.
$$

that $\widetilde{H}_{k}(j \Omega)$ is obtained from periodically extending the analog analysis filter $H_{k}(j \Omega)$ with the period $\frac{2 \pi}{T}$ in the same manner as $\widetilde{X}(j \Omega)$. The Perfect Reconstruction (PR) is accomplished when the output $\widehat{x}\left[n^{\prime}\right]$ and input samples $x\left[n^{\prime}\right]=\left.x(t)\right|_{t=n^{\prime} T}$ are the same except with a possible delay $n_{d}$ as $x\left[n^{\prime}\right]=x\left[n^{\prime}-n_{d}\right]$. The PR conditions may be interpreted by the following equations [5]:

$$
\left\{\begin{array}{l}
T_{\circ}\left(e^{j \omega}\right)=e^{-j \omega n_{d}} \\
T_{m}\left(e^{j \omega}\right)=0 \quad m=1, \ldots, M-1
\end{array}\right.
$$

\section{II-B. MIMO HFB architecture}

In the subband and TDM MIMO HFB architectures, the related parallel ADC tries to perfectly reconstruct the input vector $\mathbf{s}[n]$. To design the synthesis filters matrix $\mathbf{F}\left(e^{j \omega}\right)$, the $M$ analog analysis filters are substituted by an $M \times M$ matrix of digital filters $\mathbf{H}\left(e^{j \omega}\right)$. Each element $H_{k l}\left(e^{j \omega}\right)$ of $\mathbf{H}\left(e^{j \omega}\right)$ stands for a digital filter which is obtained from the analog analysis filter $H_{k}(j \Omega)$ depending on the type of MIMO HFB structure. For both the subband and TDM HFBs, the frequency response $\widehat{S}\left(e^{j \omega}\right)$ of the output vector $\widehat{\mathbf{s}}[n]$ may be described in terms of the input vector $S\left(e^{j \omega}\right)$ as follows:

$$
\widehat{S}\left(e^{j \omega}\right)=\mathbf{T}\left(e^{j \omega}\right) S\left(e^{j \omega}\right)=\mathbf{F}\left(e^{j \omega}\right) \mathbf{H}\left(e^{j \omega}\right) S\left(e^{j \omega}\right)
$$

where $\mathbf{T}\left(e^{j \omega}\right)$ is a matrix containing distortion and InterChannel Interference (ICI) terms. It reveals that the estimated value $\widehat{s}_{k}[n]$ of $k^{t h}$ element of input vector $\mathbf{s}[n]$ may be developed in the frequency domain as following:

$\widehat{S}_{k}\left(e^{j \omega}\right)=\underbrace{T_{k, k}\left(e^{j \omega}\right) S_{k}\left(e^{j \omega}\right)}_{\text {Distortion }}+\underbrace{\sum_{m=0, m \neq k}^{M-1} T_{k, m}\left(e^{j \omega}\right) S_{m}\left(e^{j \omega}\right)}_{I C I}$

The $(k)^{t h}$ diagonal element $T_{k k}\left(e^{j \omega}\right)$ of $\mathbf{T}\left(e^{j \omega}\right)$ stands for the distortion function related to the input component $S_{k}\left(e^{j \omega}\right)$. The other $M-1$ elements of $(k)^{t h}$ row of the $\mathbf{T}\left(e^{j \omega}\right)$ represent the relative ICI terms. The ICI elements are desired to be ideally null. Then, the PR equations at each frequency $\omega$ will be:

$$
\mathbf{F}\left(e^{j \omega}\right) \cdot \mathbf{H}\left(e^{j \omega}\right)=\mathbf{I} \cdot e^{-j \omega n_{d}}
$$

where I represents the identity matrix $(M \times M)$ and $n_{d}$ stands for an arbitrary delay. $n_{d}$ is considered for maintaining the causality. $\mathbf{H}\left(e^{j \omega}\right)$ may be obtained for the subband and TDM MIMO HFBs as following.

\section{- Subband HFB structure}

To obtain the $k^{t h}$ row of analysis filter matrix $\mathbf{H}\left(e^{j \omega}\right)$, the analog filter $H_{k}(s)$ is considered in the frequency interval $\left[-\frac{\pi}{T}, \frac{\pi}{T}\right]$. Then, $H_{k 0}(z), H_{k 1}(z), \ldots$, and $H_{k(M-1)}(z)$ are extracted in the same way that the subband input components are found from the input signal [9].

\section{- TDM HFB structure}

In the TDM case, the extraction of $\mathbf{H}\left(e^{j \omega}\right)$ from the $M$ analog analysis filters may virtually imagined as sampling the related impulse-responses. In the frequency domain, each element $H_{k r}\left(e^{j \omega}\right)$ of $\mathbf{H}\left(e^{j \omega}\right)$ may be obtained from the $k^{t h}$ analog analysis filter $H_{k}(j \Omega)$ as following [10]:

$$
H_{k r}\left(e^{j \omega}\right)=\frac{1}{M} e^{j \frac{\omega}{M} r} \sum_{m=0}^{M-1} e^{-j \frac{2 \pi}{M} r m} \widetilde{H}_{k}\left(j \frac{\omega}{M}-j \frac{2 \pi}{M} m\right)
$$

where $\widetilde{H}_{k}(j \Omega)$ stands for the periodic extension of $H_{k}(j \Omega)$ considering the interval $\left[-\frac{\pi}{T}, \frac{\pi}{T}\right]$. 


\section{DESIGN OF SYNTHESIS STAGE}

Using the PR equations (2) and (4), an HFB-based ADC may be designed provided that one of the respective synthesis or analysis filter banks are a priori known. According to the constraints of analog circuits, it is practically preferred to design the digital synthesis filters assuming a preselected set of analog circuits as analysis filters. The frequency response of synthesis filters may be obtained at each frequency $\omega$ using (2) and (4) knowing the analysis filters. Finite-Impulse Response (FIR) filters are conveniently-realizable and need only a limited resource of memory and processing. Using FIR filters, the equations would be linear in terms of the unknown coefficients of synthesis filters as well. Then, the frequency response of synthesis filters can be approximated by the FIR digital filters. The number of coefficients $L$ of each FIR synthesis filter plays an important role in determining the distortion and aliasing (or ICI for the MIMO HFB) terms.

In practice, the PR equations are incompatible at the frequencies near the spectral borders $\left( \pm \frac{\pi}{T}\right)$. To achieve a suitable resolution at the output of HFB ADC using FIR synthesis filters, these frequencies have to be neglected. For this purpose, the analog input $x(t)$ is supposed to occupy just the spectrum interval $\left[-(1-\alpha) \frac{\pi}{T},(1-\alpha) \frac{\pi}{T}\right]$ in the classical HFB case that $\alpha$ represents the oversampling ratio. The optimal oversampling ratio for an eight-branch classical HFB has been reported to be about $7 \%$. Similarly, a spectral part of each input component of MIMO HFBs has to be allocated as Guard Band (GB). In the subband case, GB covers both the low and high frequencies of each subband component. However, the GB of TDM HFBs is sufficient to accommodate either low or high frequencies at the spectrum of each TDM component depending to have an even or odd number $M$ of branches respectively [10].

\section{EVALUATION OF VARIOUS HFBS}

Using a simply-realizable class of analog circuits for the analysis filter bank and neglecting the quantization noise, an eight-branch HFB-based A/D converter is designed and simulated in this section. It is supposed that the analysis filter bank is composed of the second-order RLC circuits except one first-order RC circuit as low-pass filter. All the secondorder RLC circuits are assumed to have a constant passing band. The synthesis filters are supposed to be FIR digital filters with 64 coefficients. The results are discussed and compared for the classical, subband and TDM architectures in terms of different properties.

\section{- Sensitivity to analog imperfections}

Tables I and II list the output resolutions considering a sinusoidal and a chirp input signal respectively. It may be seen that the output resolution of TDM HFB-based ADC is much larger than the one related to the other HFB structures in the absence of realization errors of analysis filter bank. In the presence of analog imperfections, the output resolution of all HFBs reduces rapidly. Considering the realization errors, the output resolutions of TDM and subband HFB structures are nevertheless approximately 2 and 1 bit better than the one due to the classical HFB respectively. Then, the MIMO HFB architectures exhibit less sensitivity to the analog imperfections than the classical HFB. To better compare the performance of different HFB architectures, the error spectrum of outputs are illustrated in Fig. 3 for these HFB architectures. All the output components of subband HFB are zero except the first subband which is associated with the input sinusoidal signal. The error signal of the classical HFB is clearly larger than the one associated with the MIMO HFBs for this sinusoidal input.
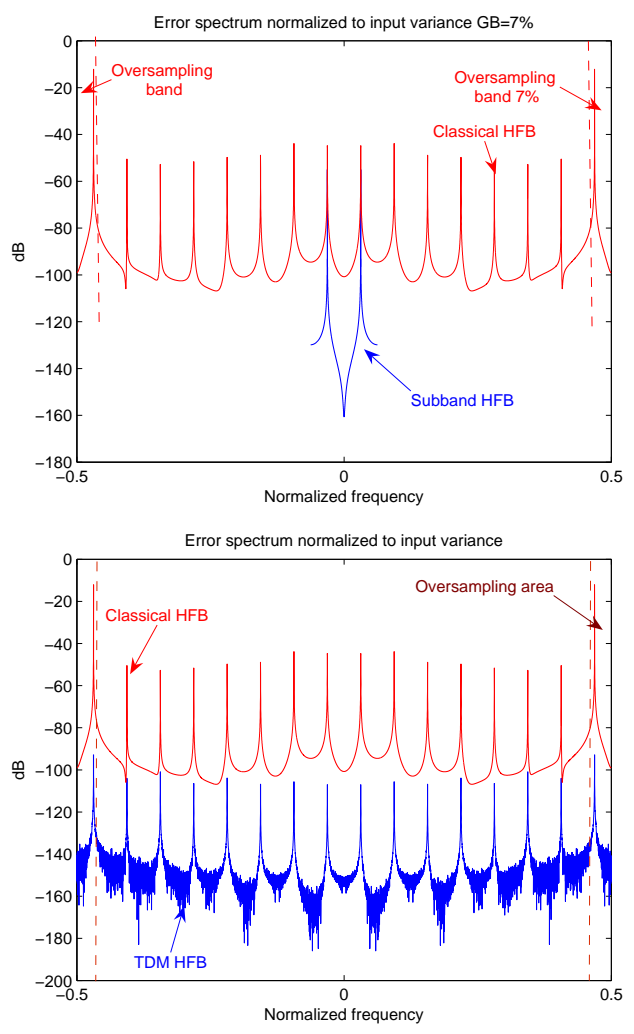

Fig. 3. The error spectrum compared with the classical HFB for the subband (top) and TDM (bottom) HFBs versus normalized frequency.

\section{- Applicability of blind techniques}

The TDM and subband HFB structures provide an LTI relationship between the related inputs and outputs in opposite to the classical HFB. The automatic noise cancelation may be applied to the TDM and subband HFBs for correcting the realization errors of analysis filter bank. However, the blind deconvolution techniques are only applicable to the TDM one. The subband structure can not exploit the blind deconvolution methods because it necessitates to consider a non-Gaussian input signal being white at both time and 
Table I. The comparison of HFB sensitivities to realization errors in terms of output resolution (in bits) considering a sinusoidal input located at the middle of first subband.

\begin{tabular}{|c|c|c|c|}
\hline \multicolumn{4}{|c|}{ Output resolution (in bits) for a sinusoidal input } \\
\hline Realization & The type of 8-branch HFB Architecture \\
\cline { 2 - 4 } errors (\%) & Classical & Subband & TDM \\
\hline $0 \%$ & 9.9 & 10.5 & 21 \\
\hline $1 \%$ & 8 & 10 & 9.8 \\
\hline $5 \%$ & 5.8 & 7 & 8 \\
\hline
\end{tabular}

Table II. Comparison of HFB sensitivities in reference to the output resolutions supposing a chirp input signal sweeping the first subband.

\begin{tabular}{|c|c|c|c|}
\hline \multicolumn{4}{|c|}{ Output resolution (in bits) for a chirp input } \\
\hline $\begin{array}{c}\text { Realization } \\
\text { errors (\%) }\end{array}$ & The type of 8-branch HFB Architecture \\
\cline { 2 - 4 } & Classical & Subband & TDM \\
\hline $0 \%$ & 9.6 & 10.1 & 17 \\
\hline $1 \%$ & 7.2 & 8.0 & 8.6 \\
\hline $5 \%$ & 5.3 & 6.1 & 6.6 \\
\hline
\end{tabular}

frequency domains. On the other hand, the whiteness at both the time and frequency domains can not simultaneously hold for the stochastic processes except the Gaussian one.

\section{- Complexity of synthesis stage}

The classical HFB-based A/D converter consists of $M$ FIR synthesis filters, but the MIMO subband and TDM architectures need $M^{2}$ ones (figure 2). For an FIR filter with $L$ coefficients, $L$ multiplying operations and delay components are effectively necessary. Then, for implementing the synthesis stage, the MIMO architecture will need $M^{2} L$ multiplications to be compared with $M L$ ones in the classical case. Nevertheless, it does not require the upsampling operations (zero-padding by $M$ ) in the MIMO case. Moreover, the MIMO HFB structure provides $M$ output samples compared with only one output sample obtained from the classical HFB at each cycle. Therefore, the computational complexity per each output sample is equivalent for both classical and MIMO HFB structures.

To thoroughly compare the computational complexity, the design phase has to be considered as well. In the design phase, FIR synthesis filters are obtained. Assuming $N$ frequency points for designing the synthesis filters, conventional HFB structure would require the inversion of an $M N \times M N$ matrix. The MIMO HFBs need invert $N$ matrices of $M \times M$. In practice, $N$ must be much larger than $M(N>>M)$ to have an acceptable interpolation. Thus, the design phase of classical HFB architecture is computationally much more complex than the subband one. The complexity of the design phase is particularly important when an adaptive method is applied to estimate the real analysis filter bank for compensating realization errors.

\section{CONCLUSION}

The HFB-based ADCs are discussed in this paper as a good candidate for realizing the SDR approach. Two MIMO architectures called subabnd and TDM HFBs are introduced and the relative PR equations are described as well as the related design method. Simulating both classical and MIMO HFBs in the time-domain, the MIMO HFB-based ADC appears less sensitive to the errors of analog analysis filters regarding to the output resolution. The computational complexity per each output sample is the same for both conventional and MIMO HFBs. At last, the MIMO HFBs may be corrected using noise canceling technique, although the blind deconvoultion methods are applicable only to the TDM one. Applying a blind estimation algorithm, the sensitivity of HFB structure would be reduced so that a wideband ADC may practically be obtained.

\section{REFERENCES}

[1] Petraglia, A. and Mitra, S. K., "High-speed A/D conversion incorporating a QMF bank", IEEE Trans. on Instrumentation and Measurement, vol.41, no.4, June, 1992, pp.427-431.

[2] Zhao, S. et al. "A parallel ADC structure for software radio receiver", Proc. Conf. on Convergent Technologies for Asia-Pacific Region, Oct., 2003, pp.1025-1027.

[3] Tuttlebee W., Manor R., "The Software radio technology: a european perspective", IEEE Comm. Mag., Feb., 1999, pp.118-123.

[4] Velazquez, S.R. et al. "Design of Hybrid Filter Banks for Analog/Digital conversion", IEEE Trans. on Sig. Proc., vol.46, no.4, Apr., 1998, pp.956-967.

[5] Lowenborg P., et al. "Two-channel digital and hybrid Analog/Digital multirate filter banks with very lowcomplexity analysis or synthesis filters", IEEE Trans. on circuits and Systems, vol.50, no.7, July, 2003, pp.355367.

[6] Asemani D., Oksman J., "Influences of oversampling and analog imperfections on hybrid filter bank A/D converters", IEEE MWSCAS, San Juan, PR, Aug., 2006.

[7] Sanada Y., Ikehara M., "Digital compensation scheme for coefficient errors of complex filter bank parallel A/D converter in low-IF receivers", Proc. IEEE Vehicular Technology Conf., vol.4, May, 2001, pp.1680-1684.

[8] Guilloud, F. et al. "Etude d'un algorithme itratif de repliement spectral lors d'une conversion $\mathrm{A} / \mathrm{N}$ parralle ", Proc. Journees Nationales du Reseau Doctoral de Microelectronique, Apr., 2001.

[9] Asemani D., Oksman J., "Performance of subband HFBbased ADC", Proc. ISSPA, Sharjah, UAE, Feb., 2007.

[10] Asemani D., Oksman J., "TDM architecture for Hybrid Filter Bank A/D converters", submitted to IEEE MWSCAS, Montreal, Canada, Aug., 2007. 